\title{
Performance Comparison Between a Statistical Model, a Deterministic Model, and an Artificial Neural Network Model for Predicting Damage From Pitting Corrosion
}

M. Urquidi-Macdonald and D. D. Macdonald

Pennsylvania State University, University Park, PA 16802

\begin{abstract}
Varinus attempts have been made to develop models for predicting the development of damaye in metals and alloys duc to pitting corrosion. These mudels may be divided into two classes: the empirical approach which employs extremc value statistics, and the detcr. ministic approach based on perceived moclaanisms for nucleation and growth of damage. More reecntly, Artificial Neural Nerworks (ANNs), a mondeterministic lype of motel, has becn deve!oped to describe the progression of damage due to pitting cortusion. We compare the three approactics abuvestatistical, deterministic, and neural networks. Our goal is to illustratc the
\end{abstract}

advantayes and disadvantages of each approach, in order that the most reljable methods may be employed in fus. ture algorithms for predicting pitting damuge functions for enginecring structures. To illusirate the difficulty that we face in predicting cumulative pitting damage, we selected a set of data lhat was collected in the laboratory. Wc cumpare and contrast the three approaches by referenee to this dala set.

Key words: artificial neural nctworks; detcrministic; mathemutical modeling; pitting corrosing; statistics.

Accepted: March 22, 1994

\section{Introduction}

On the basis of laboratory studies [1], and through the analysis of field data collected over the past decade by Battelle Columbus Laboratory [2], several factors have been identified as contributing to the development of pitting damage in gas fired heat exchangers in domestic and industrial service:

(i) The type of alloy used for fabricating the heat exchanger

(ii) Chloride concentration in the flue gas condensate

(iii) Temperature

(iv) Exposure time

(v) Ambient versus indoor air

(vi) $\mathrm{pH}$

(vii) Electrochemical potential

Unfortunately, few of these factors are simply related to the damage functions or to one another.
Accordingly, it is seldom possible to establish a simple empirical equation for predicting pitting damage as a function of these variables. The case cited above is not atypical, and it illustrates the difficulties faced by those who seek to develop predictive models for assessing corrosion damage. Indeed, the data base established by Battelle is probably one of the best that currently exists for the development of pitting damage in an industrial system. A full interpretation of the Battelle data in terms of statistical, deterministic, and artificial neural network models is published elsewhere [3].

In the present paper, we use a more restricted databuse to illustrate how various classes of models arc used to analyze the damage caused by pitting corrosion. These models include a statistical approach based on the Weibull distribution function, a deterministic model based on a physicochemical 
mechanism, and an Artificial Neural Network (ANN) that assumes neither a mathematical mode? nor a physical model, but which seeks to establish relationships between the dependent and indepertdent variables by examining the patterns contained within the data set.

\section{Experimental Data}

We used laboratory data to illustrate the time and potential dependencies of pitting damage. To do this, we chose a laboratory data set for which the following independent variables were identified: 1) concentration of minor alloy elements in weight percentages, 2) difference in oxidation state between host metal and minor alloy elements, 3) applied potential, and 4) time of observation. Independent variables 1) and 2) are related to the type of alloy; and independent variable 4) together with solution composition (which was maintained constant) is determined by the electrochemical potential. Temperature, solution composition, and $\mathrm{pH}$ were maintained constant. The dependent variable was the total number of pits.

We then used this set to illustrate the prediction of cumulative damage for pitting corrosion using three different models: statistical, deterministic, and artificial neural networks. The data were measured by English and Macdonald at SRI International [1].

Several alloys of nickel were fabricated. Each of the alloys tested was arc-melted from powders under an Argon gas blanket in a sealed container. Binary nickel alloys containing $\mathrm{Al}, \mathrm{Ta}$, and $\mathrm{Mo}$ in nominal concentrations of $0.1 \%, 0.5 \%, 1 \%, 3 \%$, $5 \%$, and $8 \%$ by weight were cast as $100 \mathrm{~g}$ buttons and were sectioned in an acrylic plastic hefore polishing. The alloying elements were selected on the basis of their oxidation states relative to nickel (oxidation state $=2$ ). The excess oxidation statcs range from 1 for Al to 4 for Mo.

The polished specimens were placed in a cell. The electrode potential was swept in the positive direction at $1 \mathrm{mV} / \mathrm{s}$ from an initial potential of 0.0 $\mathrm{V}$. This results in a distribution in breakdown potentials. Alternatively, the potential was stepped from $0.0 \mathrm{~V}$ to $0.325 \mathrm{~V}, 0.375 \mathrm{~V}, 0.4 \mathrm{~V}$, and $0.45 \mathrm{~V}$. This resulted in a distribution in induction (or observation) times for the nucleation of pits.

In both types of experiments the pit nucleation and growth events were photographed at $65 \times$ magnification at regular intervals. The number of pits were counted on the pictures tiken at different times and conditions.
The pitting data were measured scveral times on a similar sample to explore reproducibility. The reproducibility in pure nickel appeared satisfactory (about $10 \%$ difference between runs), but the reproducibility from alloy composition to alloy composition was different. Rcproducibility was better at high potentials perhaps because the total number of pits developed was higher. Reproducibility appeared to be better at high minor alloy contents and high oxidation states (about $20 \%$ ), than at low minor alloy contents and low oxidation states (about $50 \%$ ). Regardless of the poor reproducibility in some of the samples, a general trend was observed: a) The cumulative number of pits diminishes with 1) minor alloy element content, and 2) with increasing difference in oxidation state between the base alloy and the minor alloy element; and $b$ ) The cumulative number of pits grows with increasing applied potential and observation time.

Cumulative pitting damage is an irreversible, dynamic, time decay, environmentally related process. The literature is abundant in pitting corrosion data, but there is a lack of good quality data be. cause of the dirficulty of measuring pitting corrosion when controlling all the environmental parameters.

All model building is concerned with an attempt to increase our knowledge of complex physical reality. The parameters plus the validity of the model must be determined from the data. The philosophy behind the type of model is different. The information obtained from a purely probabilistic model (statistic and stochastic models) is about finding embodied in the data trends that can be used in future predictions. The information obtained from a deterministic model is about the physical meaning of the phenomena itself. The information obtained with a ANN nodel is about the dependency and importance of input /output relationships. In any case, the model capabilities need to be tested.

We can start the process of solving our problem hy list ing facts, listing observations, and fisting existing laws relating variables and outputs. Then we have to ask ourselves which will be the best model to describe the problem, and what do we expect from the model. Later we need to identify the model or models to use; specify the constraints, choose the coordinates, and apply the laws dictated by the model. Important questions related to the choice of a correct model are: Is the process static or dynamic? Is the process stationary or not?; Are the available data distributed or not? What do the data mean"? What is the data variance? What are the correlations?. In any case, the fitted model you 
use for analyzing your data is the nefrest rejutson tation of the true situation you have availabls.

\section{Statistical Approach and Results}

Stochastic processes are dynamic, and gond ix amples are fatigue, wear, and crack ur pit grow $1 \mathrm{~h}$ There are two main types of stochastic processes: stationary and nonstationary.

It is well known from experimental data that cumulative pitting damage is a nonstationary phenomenon. It is well known that nonstatiunary models and their estimation are notoriously difficult problems to handle except for special cuses. Discrete state continuous Markoff processus are good examples of models that describe nonstatiunary stochastic phenomena. However, there is no literature on problem solving using nonstationaly finite Markoff chains [4]. On the other hand, for the last data set [1] (measured at the laboratory), Ilse cumulative damage versus time was meisured, lout the pit depth versus number of pits was not. There.fore, it is impossible to derive a dynamic model for pitting damage using that data set. The only option available is to try to fit a static model (i.e., our hy. pothesis is that the numbers of pits versus pit depth does not change with time). We choose a 2 parameter Weibull distribution; for which we assume that the independent parameters are potential, allu the oxidation state and concentration of the minur alloying elements. The dependent variables are the cumulative number of pits and the induction time. The Weibull distribution function is

$$
F(x)=\left(1-\exp \left(-(x / \beta)^{\mathrm{in}}\right)\right)
$$

where $\alpha$ and $\beta$ are fitting parameters and $x$ is the dependent variable of interest.

We normalized the data set $1080 \%$ of its nuximum value, allowing $20 \%$ of the pits to nucletste if the time would have been extended to infinity. For each potential, oxidation state, and percentile of minor alloy element, we performed a nonlinear fit to estimate the Weibull fitting parameters.

The choice of a Weibull distribution is arlintary, we chose a Weibull distribution instead of sime other probabilistic distribution because of the flexibility that this distribution offers in fitting diflierunt shapes obtained when plotting cumulative damage versus dependent varjables.

The nonlinear fits were acceptable (sum of square errors between fit and data $<20 \%$ for w w $\beta$ ). We used those data sets for which smooth changes of $\alpha$ and $\beta$ were calculated as it function of

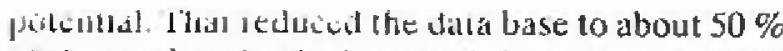
of Ite lolal al ailable (the tolal data base had 1400 data limes comlaining number of pils at different obsonaliun times, applied potential, oxidation states, and percentule of minor elensents). We plotted the $a$ and $\beta$ values as a function of potential. Figures la alld lu stiow the iesulss. The beta parameter of the Weibull alistsibution appears to not change with applicu futcutial at high concentration of minor alloy elements (5\%), but it changes drastically with applied potenrial at luw concentrations of minor alloy eitement $(3 \%, 1 \%)$. We the fit polynomials describing $\alpha$ and $\beta$ is functions of applied potential, oxidations slate, and percentile of minor alloy elements.

Whe Werbull distribujon with $\alpha$ and $\beta$ as paramelets was used to generate the cumulative damage function Fignes $2 a, b$, and $c$ show the predictions vbtained whth this siutislical model. When we compared the predictions sbrained with this model and the measured data, we olserved that both trends are similar. Huwever", it would be very risky to use the mudel to miahe predictions for other oxidations states, pirtentile of minor alloys elements, or applied purentials outside the range for which the Weibull-panametars were calculated.

It is well known that the Weibull distribution is a sufliciently flexilble function that practically any set of dald cath be tille, by it. However, the problem we fated is that we du llut know a priori the correct relationshps betwen $\alpha$ and $\beta$ and the independent variables.

Predictions wilh the sank nudel for oxidation stales grealer than 32 gave cumulative probability wl ceru at aly" linte and are not shown. The designativin " 3 2" referes to the oxidation state of the alloying telenient $(\mathrm{Al}=3$ ) and the host inetal $(\mathrm{Nj}=2)$.

\section{t. Deterininistic Model}

A cunjilècly successfol mudel must account for all of the phenomenological correlations that exist tictwesl pinting susceptibility and pit velocity, and various tnvirommental and electrochemical factors, such as lempelature, $\mu \mathrm{H},[\mathrm{CJ}]$, potential, time, and alluy composition. "The Point Defect Model (PDM) $[5,6]$ accounts for the effects of clectrochemical poteritial, alliy composition, and environmental conditions on the nucleation of pits.

The determmistic model is based on the PDM and the Solute Vacuncy Interaction Model (SVIM) [7-10]. The PDM proposes that passivity breakdowit vecurs because of an enhanced flux of cation vacancics from the filmisolution to the metal/film 

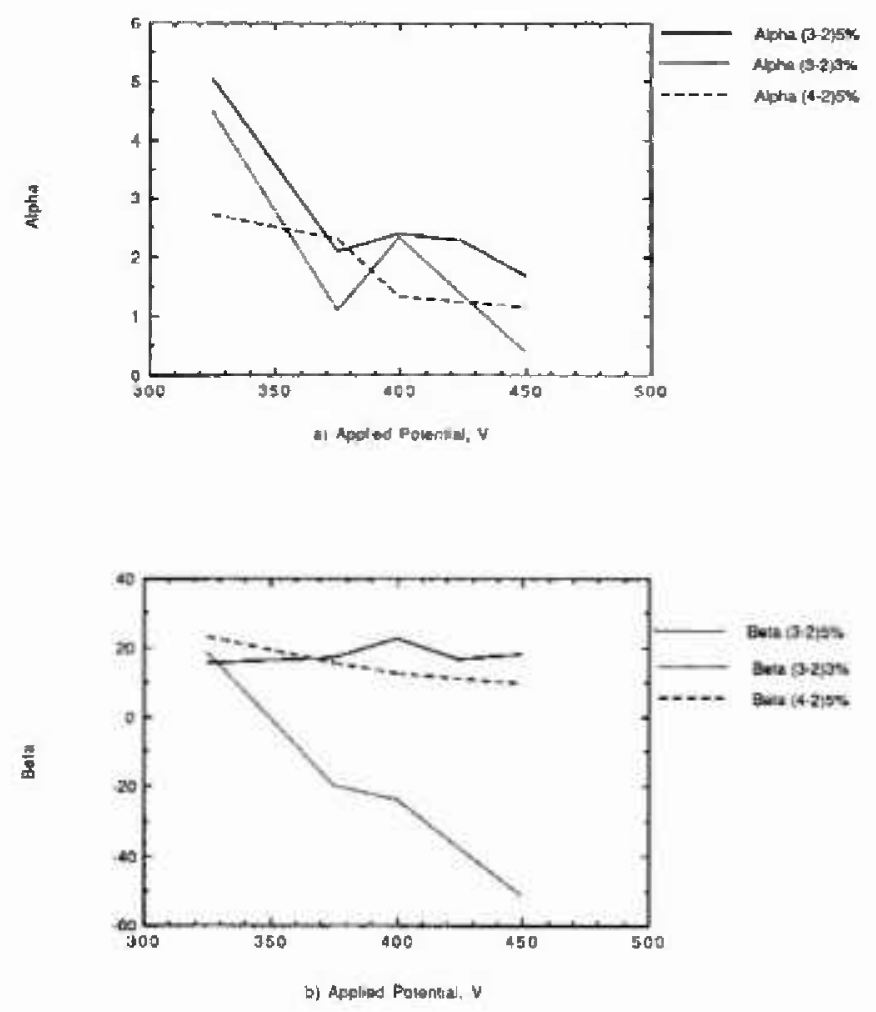

Fig. 1. (a) Aipha parameter of the Weibull distribution versus applied potential; at several percentiles of minor alloying elements, and oxidalion states. (b) Beta parameter of the Wcibull distribution versus applied potential; at several percentiles of minor alloying ele. ments, and oxidation states.

interface. If the excess of vacancies arriving at the interface between the metal and the film can not be absorbed into the metal or be annihilated by some appropriate mechanism at high enough rate, they accumulate to form a vacancy condensate at the metal film/interface, which then grows to a critical size. The PDM is used to calculate the breakdown potential and induction time. The effect of the minor alloying elements in the oxide film on the breakdown parameters is modeled using the SVIM. The SVIM is based on the hypothesis that highly ox. idized solutes in the passive film electrostatically complex with the mobile cation vacancies.

The PDM and SVIM results in distributed values of the breakdown potential and induction time, and complexing between the immobile alloying element in the film (the "solute") and the mobile vacancies diminishes the flux of vacancies across the film. This leads to an increase in the breakdown potential and the induction time for film breakdown. The higher the net oxidation state (minor alloy element oxidation-host ion oxidation) and/or the higher the percentile of minor alloying elements in the film, the greater the effect on reducing the flux of vacancies and hence in increasing the pitting potential and the induction time. Once the pits nucleate, they grow at different rates. To calculate the pit growth rate we used 1) a simplistic steady state model suggested by Alkire [11]; and 2) a nonstationary model developed by us [12-13]. The stationary model is expected to be adequate for only short times.

The overall model (combination of the PDM, SVIM, and pit growth) requires the defining of a number of parameters, as shown in Table $I$.

Figure 3a shows the cumulative probability of the number of pits (normalized to 1 ) as a function of pit depth and observational time of $50 \mathrm{~s}$, for an applied voltage of $0.325 \mathrm{~V}$ and for several concentrations of the minor alloying element with oxidation state of 3-2 (example aluminum in nickel). Figures $3 \mathrm{~b}$ and $3 c$ show similar plots for oxidation states of 4-2 (e.g., titanium-nickel) and 6-2 (e.g., molybdenumnickel), respectively, for the same conditions. It is interesting to note the great effect of minor alloying elements with high oxidation states. The model predicts that the cumulative probability of the number 

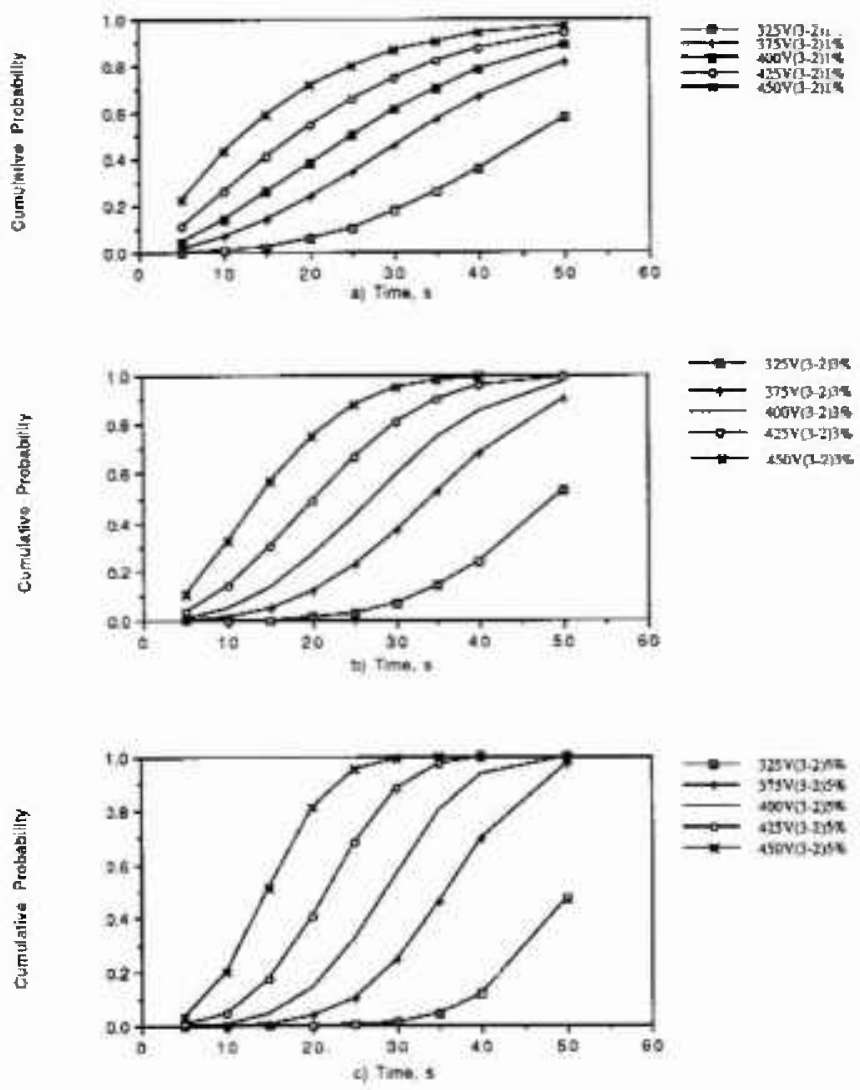

Fig. 2. Predicted cumulative probability, obtained using the statistical model, versus time of obscrvation, at several applied potiontials. (a) Oxidation state 3-2; and $1 \%$ of minor alloying clement segregaled in the film. (b) Oxidation stote 3-2; and $3 \%$ of minor alloying element segregated in the film. (c) Oxidation state $3-2$; and $5 \%$ of minor alloying element segregated in the film.

Table 1. Input data used in the calculation of the detcrministic/probabilistic model

\begin{tabular}{|c|c|c|}
\hline Parameters & Value & Units \\
\hline Stoichiometry & 2 & \\
\hline Avogrado constant & $6.023 \mathrm{E}+23$ & moll-1 \\
\hline Mol vol. of oxide cation & 30 & $\mathrm{~cm}^{3} / \mathrm{mol}$ \\
\hline Gibbs energy change & $-40,0000$ & $\mathrm{~J} / \mathrm{mol}$ \\
\hline Gibbs encrgy change & $-10,600$ & $\mathrm{~J} / \mathrm{mol}$ \\
\hline Mean diffusion coefficient & $5 \mathrm{E}-20$ & $\mathrm{~cm}^{3} / \mathrm{s}$ \\
\hline Standard deviation & 0.75 Dmcan & $\mathrm{cm}^{2} / \mathrm{mol}$ \\
\hline Chloride activity & $0.573 / 2$ & \\
\hline Electrical field across film & $1.1 \mathbf{E}+6$ & $\mathrm{~V} / \mathrm{cm}$ \\
\hline Alpha & 0.65 & \\
\hline Beta & -0.01 & $\mathrm{~V} / \mathrm{pH}$ unit \\
\hline Critjeal area vacancy size" & $1 E+16$ & $\mathrm{No} / \mathrm{cm}^{2}$ \\
\hline Critical vacancy flux & $15.87 \mathrm{E}+12$ & $\mathrm{No} / \mathrm{cm}^{2+}$ \\
\hline Temperature & 298.15 & $\mathrm{k}$ \\
\hline Applied potential & -0.55 & V SIIE \\
\hline Molar gas constant & 8.314 & $\mathrm{~J} \mathrm{~K}^{-1} \mathrm{mul}^{-1}$ \\
\hline Electrical putential film/sol & -0.5 & V SHE \\
\hline
\end{tabular}

"Variables that were used to adjust one datum point to seale the results properly. 


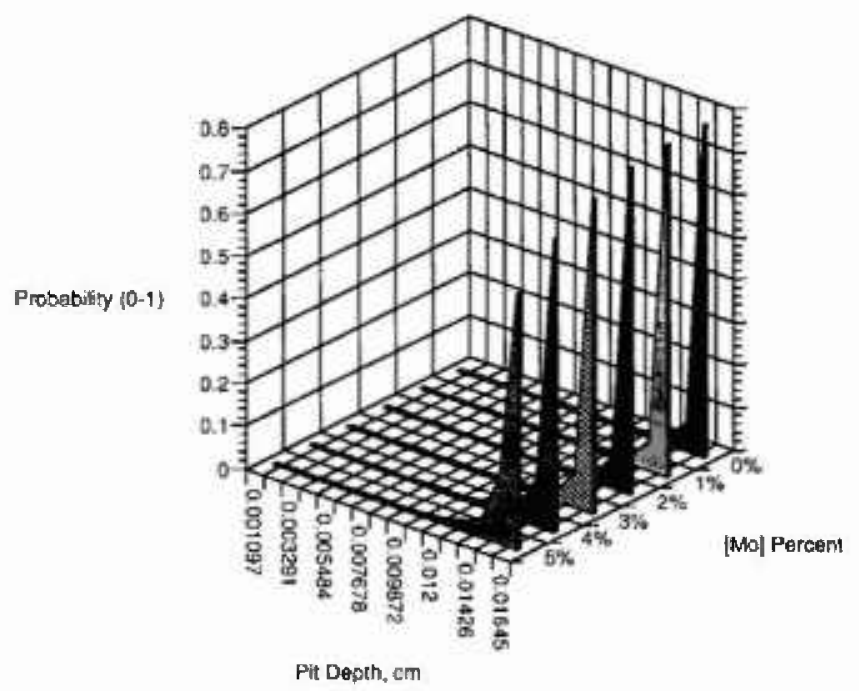

Fig. 3a. Cumulative probability, calculated using the deterministic model, versus pit depth for scveral concentratjons of minor allnying element segregated in the film for oxidation state (3-2).

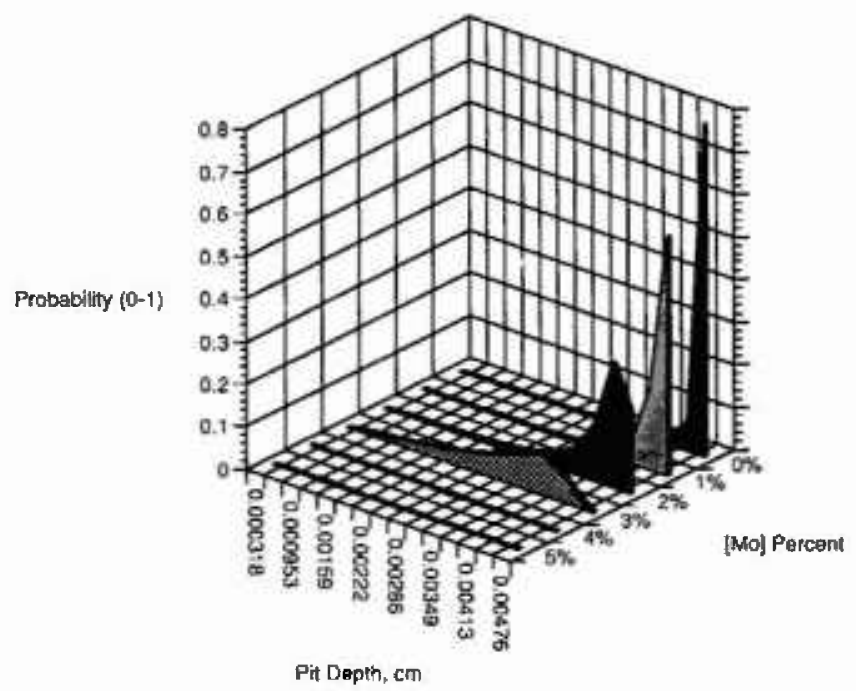

Fig. 3h, Cumulative probability, culculatcd using the detcrninistic model, versus pit depth for several coneentrations of minor alloying clement segregated in the film for oxidation state (4-2). 


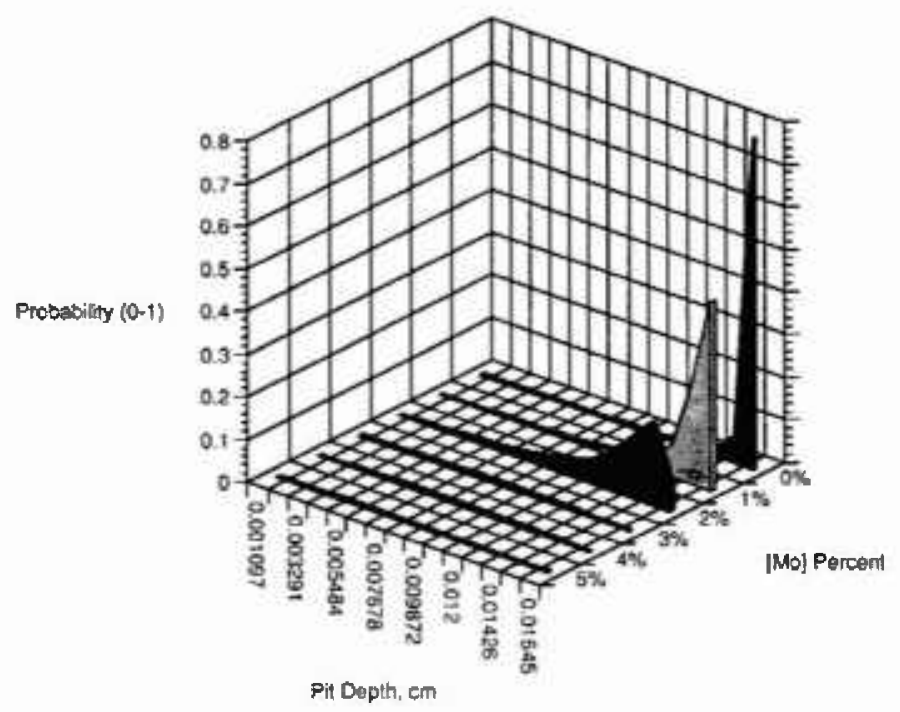

Fig. 3c. Cumulative probability, calculated using the deterministic model, versus pit depth for several concentrations of minor alloying element segregated in the film for oxidation state $(6-2)$.

of pits at all pit depths is higher at lower minor alloying element oxidation state and at lower concentration of the minor alloying element. Because the model does not assume a total number of breakdown sites only a normalized probability is obtained.

Figure 4 shows the beneficial effect of adding minor alloying elements with high oxidation states.

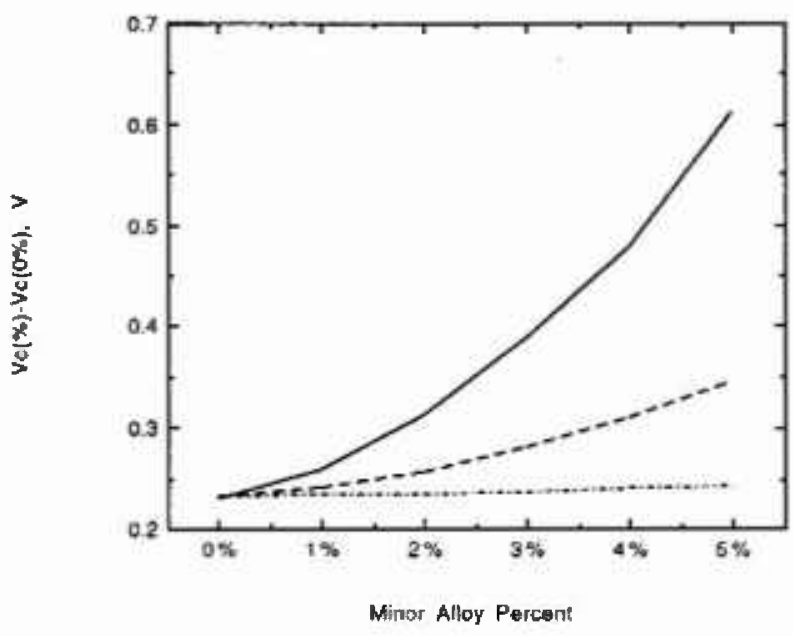

Fig. 4. Difference between calculated breakdown potential of nickcl containing $0 \%-5 \%$ of minor alloying elements with oxidation states of ( 3-2: Ni-Al), (-- 4-2: Ti-Ni), and (- . . - .6-2: Mo-Ni) and calculated breakdown potental of pure nickel (containing $0 \%$ of minor alloying clcments).
The breakdown potential is shifted in the positive direction, indicating that higher potentials are necessary to achieve the same damage.

\section{Artificial Neural Network Model and Results}

Probably the most efficient method, when data are available, of establishing relationships between inputs and results is to use artificial intelligence techniques. Accordingly, we describe here an Artificial Neural Network (ANN) for predicting pitting damage functions for condensing heat exchangers. When the net is trained with reliable data and knowledge, we are able to accurately predict damage outside the ranges of the input variables.

An ANN is a highly interconnected system inspired by the brain and formed by simulated "neurons" represented by a transfer function, and "weights" associated to the connections of the "ncurons." The back propagation training algorithm allows experimental acquisition of input/ oulput mapping knowledge within multilayer networks. Because we have experimental data on the cumulative numbers of pits versus time of observation, as a function of oxidation state, minor alloying element, and applied potential, we decided to use an ANN backward propagation technique with supervised learning. During training of the ANN, the cumulative numbers of pits were used as "output" and the applied potential, oxidation state, 
minor alloying element concentration, and time of observation as "inputs." We explored several topologies to obtain the best compromise between learning and computing time for an ANN with 2 hidden layers.

The maximum training time was set to 12 hours on a Macintosh II microcomputer with a threshold of $10 \%$ of the normalized input values (inputouput) [2].

The ANN had the following features:

(i) Heteroassociative memory, for which the patterns on recall from the memory are purposely different from the input pattern, because the inputs and outputs are different and belong to different classes of information.

(ii) Delta rule type of learning, where the neuron weights are modified to reduce the difference between the desired output and the actual output of the processed element. The weights are changed in proportion to the error calculated. This rule also limits the learning, if the error at the output of the network is lower than a given threshold. The learning rates of those layers close to the output are set lower than the learning rates of the other layers.

(iii) A momentum term, which is used to smooth out the changes.

(iv) A sigmoid transfer function, which is a monotonically continuous mapping function.

The ANN predictions are in good agreement with the measured data. Figure 5 shows that correlation. Considering the difficulty of obtaining high quality data, we consider that the correlation factor is satisfactory.

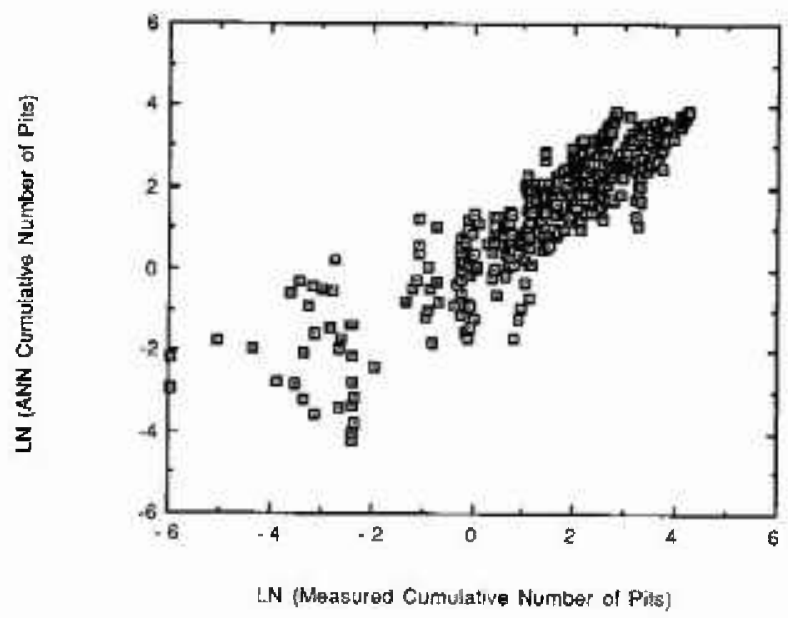

Fig. 5. Natural logarilsm of the predicted ANN total number of pits versus natural logarithm of laboratory measured totsl number of pits. The measurements included several: applied potentials, observational times, oxidation states, and pereent of minor alloying elẹments.
After the ANN was trained, it was used to make predictions of the number of pits at different applied potentials, observation times, oxidation states, and percentages of minor alloying elements in the film. The total number of pits predicted by the ANN decreased with increasing percentage of minor alloying elements in the film, and with increasing oxidation state of the alloying element (Figs. 6a and $6 \mathrm{~b}$ ). Behavior similar to that predicted by ANN was observed experimentally.

The ANN, once trained, can be used to explore the importance of the relationship between "output" and "inputs." We found that the results were strongly dependent on observation time $\left(t^{3}, t\right)$, have a medium dependency on applied potential $\left(V^{1 / 2}\right)$, and show weak dependencies on oxidation state $\left(Z^{1 / 5}\right)$ and concentration of minor alloying element in the film $\left([\%]^{1 / 4}\right)$.
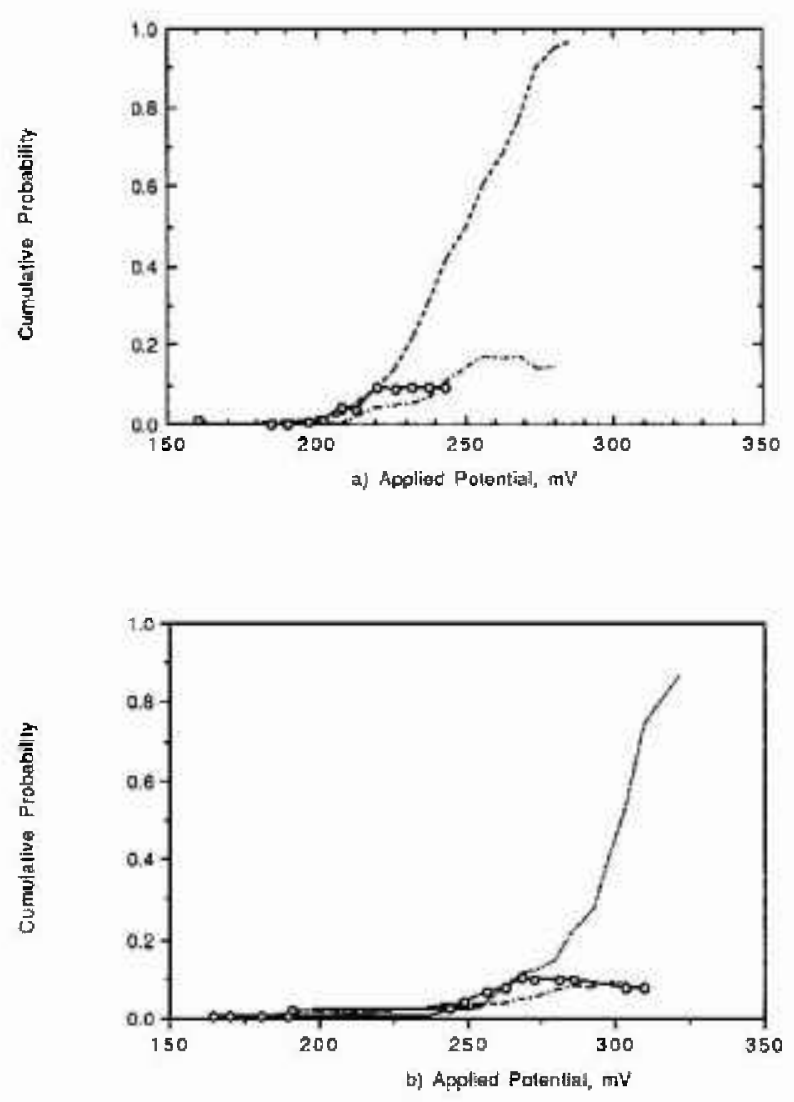

Fig. 6. ANN prediction of cumulative number of pits versus ap. plicd potential, at $50 \mathrm{~s}$ time of ubservalion; and scveral percentiles of minor alloying elements. (6a) Oxidation state (4-2). (6b) Oxidation state (6-2). 


\section{Discussion}

Figures $7 \mathrm{a}$ to $7 \mathrm{~d}$ show the best predictions obtained with the three models compared with the laboratory data.

The deterministic model predicts that the cumulative probability at low applied potentials is not only described by a flat curve but that the curve is displaced to higher times. This prediction coincides with the experimental observations. The predictions with the deterministic model at high potentials indicated that the plateau corresponding to higher times is reached sooner than that measured. The deterministic model is the only model (compared to the other two models) that brings together an understanding of the problem as well a predictive tool. Another advantage that the deterministic model has over any nondeterministic model is that to fit the model, only an experimental datum point is necessary to calibrate the model to the data. The deterministic model was developed to predict damage and cumulative number of pits simultaneously. This last capahility makes it very attractive to the user.

The results obtained with the probabilistic model are in general agreement with the experimental observations. As with the deterministic model, the plateau in cumulative damage is reached sooner than the measured one. However, the curves are flatter at lower potentials than at high potentials, but they are not displaced to higher times. The probabilistic approach needs a large data base, and the predictive capabilitics are limited to the ranges of variables confined in the data base.

The ANN model describes the cumulative number of pits very close to the experimental measurements. The plateaus on cumulative damage correspond very well to the plateau obtained experimentally. The $\triangle N N$ predictions at low number of pits are inaccurate, but they are very close to the experimental observation at higher cumulative numbers of pits.
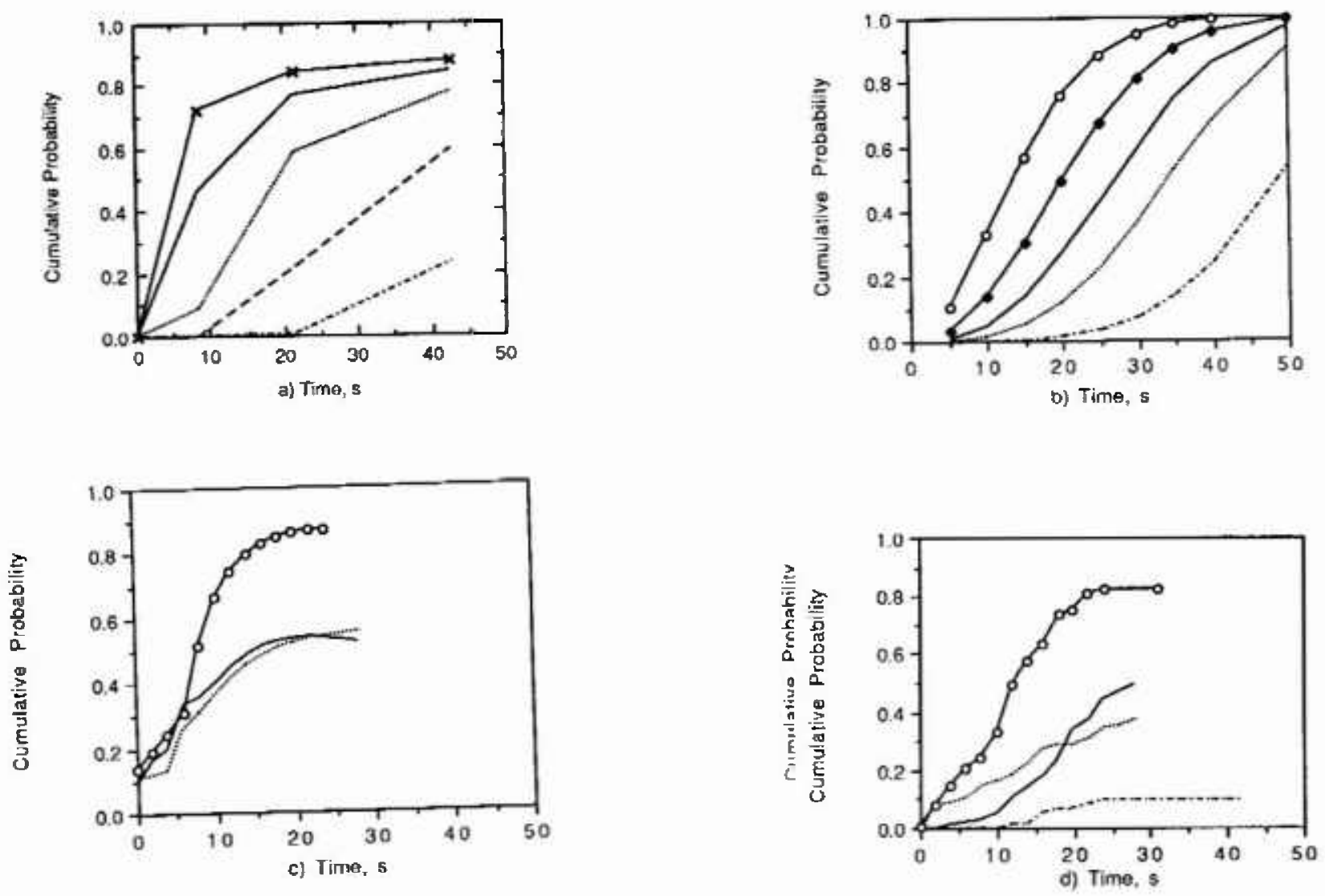

Fig. 7. Cumulative number of pits versus time of observation at several applicd potentials; Oxidutisn state (3.2); and $3 \%$ of minor alloying element. (7a) Predictions using the deterministic model. (7b) Predictions using Wcibull Distribution model with 2 fitting parameters. (7c) Predictions using the ANN. (7d) Laboratory mcasurements. 
We conclude that linle is learned athoul the phe. nomena when nondeterminiclic morlels are lised: however, they can represent invalualle oreslirtive tools. The statistical molel is in a semse mure de manding that an ANN model. It regrisos a nolust and large data base. We found that an AIN rom learn from "noisy" data, and that the range ru fro diction can be extender outside the range fort which it was trained, if trained correctly [13]. Damage functions can also be calculated usiag the cletermin. istic model based on the PDM and SVIM. Thr ile terministic model does not need to lave nn extensive data hase that includes pit depth distritu. tions. On the other band, the nondeterminisite models need a large data hase. and they arr unte to make predictions of partial damage if the pit depth versus number of pits is not conlined in the data base. Cumulative damage can he interpolated and extrapolated to other voltages and times, and is other applied potentials, for any of the three midlels. In general the results ohtained with the threc models were found to be in reasmonte apremment with experimental data [12].

We do not intend to emphasize here the impor tance of deterministic models over muleterministic models, but we have to keep in mimal. when picking a model, to choose the ome that hesl velut. sents the observations and that is rensmualy a asy in solve. Clearly, the reliability of the pvtrumlation, in particular, depends critieally on the grality of the data and on the verncity of the moslel

\section{Acknowledgments}

The authors gratefully acknowindge the suppurt of this work by the Department art lineag Hosic Energy Sciences.

\section{References}

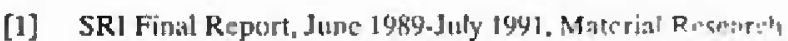
Laboratory, SRI Internatiomal, Mrunte liark, C'A.

[2] R. Razgaitis, J. H. Paver, \$, G. Talthert, F. llituliu, $\mathbf{t}$ I. White, D. W. Luxklin, R. A. Cudinik, and C. ll. shithord, Condensing Heat Exchanger for Residnutialderenmercial

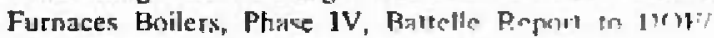
BNL, BNL Report No. 5194

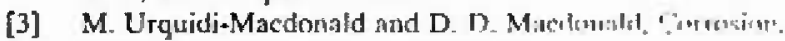
to be strbmitted for publication (1094).

[4] J. L. Bogdanuff and F. Kuzin, Frebabilistic Martals of Cumulative Damage, John Wilev \& Son (19, (10)

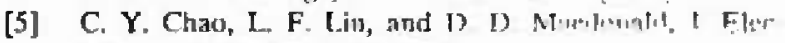
trochem. Suc. 128,1187 (1981)

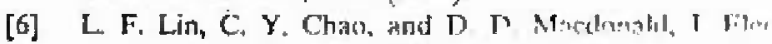
1rochem. Suc. 128, 1104 (1981). [.i) U. D. Macdmald and M. Urquidi-Macounald, Electrathem. Acta 31, 11079 (1986).

[k] A. Mrepridi-Mactomalet and D. D. Macdonald, J. Elec-

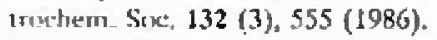

[U] Mrquidi-Macdnnald and D. D. Macdonald, J. Electritchem. Snc. 132, 555 (1985),

IJil IS. D. Machonald, M. Urquidi-Macdonald, and S. Lenhart, (1089).

[I1] I. R keck and R. C. Alkire. J. Electruchemical Soc. 126, $1652(1979)$.

[12] D. D. Macdunald and M. Urquidi-Macdonald, Corrosion $46,380(1990)$.

[(?) M. Uţundi-Macdunald, unpublishcd data, Engineering Seiener and Muchanics, Pennsylvania State University (10u?)

About the authors: M. Urguidi-Macdonald is an assorinted professor at the department of Engineering Scionce and Mechanics of Penn State. Her primary interess are in mathematical modeling of damage and life prediction. D. D. Macdonald is the Director of Penn State's Center for Advanced Materials and is a Profeswor of Materials Science and Engineering. One of his principal research interests is the development of detriministic models for predicting damage due to localized contosion. 